\title{
APPLICATIONS ENGINEERING APPROACH TO MAXWELL AND OTHER MATHEMATICALLY INTENSE PROBLEMS
}

\author{
IEEE Petroleum \& Chemical Industry Conference \\ New Orleans, LA 2002 \\ Marcus O. Durham, PhD, PE \\ Fellow, IEEE \\ Robert A. Durham, P.E. \\ Member, IEEE \\ $D^{2}$ Technologies \\ THEWAY Corp. \\ 3824 W. Ft. Worth St. \\ PO Box 33124 \\ Tulsa, OK 74153 \\ Broken Arrow, OK 74012 \\ USA \\ USA \\ Karen D. Durham \\ Member, NSPE \\ NATCO \\ 10910 E. $55^{\text {th }}$ Place \\ Tulsa, OK 74146 \\ USA
}

\begin{abstract}
Electrical engineering and physics are taught with very complex concepts involving intense mathematical manipulation. When most engineers practice, very little of the intricate science is used. The majority of problems are solved with little more than algebra. Why is there such disparity between the theory and the applications? The paper reduces all electrical theory to two very fundamental equations. A single unified equation is presented for the circuit problem. The equation is then enhanced with volumetric, motive, and lever distances. The result is a single equation for electromagnetic fields. This one equation encompasses all the fields problems including Maxwell's suite of four equations. The mathematical manipulation is never more complex than vector algebra. The procedures and considerations are items of interest to any engineer who is involved with circuits, fields, or who reads technical journals.
\end{abstract}

\section{INTRODUCTION APPLICATIONS ENGINEERS DON'T DO HAIRY MATH}

Would you agree most applications engineers are very results oriented and generally solve most of our problems without getting involved in the complex theory of the mathematics? How many times have you picked up an engineering article that was full of calculus equations and read the article in detail? [1] Can you remember Maxwell's equations, much less how to solve a del or curl? No? Then this article is for you.

After many years of blending real world problems with industrial courses, we have developed a systematic approach to the fundamental electrical problems and theory that does not use calculus or Maxwell's equations. This is a straightforward relationship. Even the most complex magnetic fields problems in traditional classroom approaches to electrical engineering simply become vectors that are resolved with linear algebra by applications engineers.

Just by changing perspective, the problem becomes much less complex. From one very simple concept, you can obtain Kirchhoff's voltage and current laws, power calculations, magnetic properties, and even Maxwell's equations, if you should ever want to do that.

There is one small detail; it will be necessary to know how to multiply an area by a distance to obtain volume. In one small step, it is possible to comprehend and see the relationship with virtually every electrical and magnetic problem.

This gives a basis for reading over those otherwise intense articles and realizing you can really comprehend what is going on without even bothering with the complex mathematics. After all, that is the approach that practical applications engineers want, isn't it?

\section{WATCH YOUR P's \& Q'S}

Rather than start with a bunch of equations, we will setout with the basic building blocks of science. One of the universal fundamentals is that every physical system develops in a group of three. This is called the triad principle, which states: Any item that can be uniquely identified can be further explained by three members. [2,3]

The three basic elements of matter are mass $(\mathrm{m})$, charge $(q)$, and magnetic pole strength or flux (p). [4,5] For the electromagnetic world, this reduces primarily to charge $(q)$ and magnetic poles ( $p$ ). Already electrical engineers have an advantage over other engineering disciplines since we cover two-thirds of the physical spectrum.

The other disciplines depend heavily on the senses to understand their concepts. You cannot see, hear, taste, smell, or touch (but one time) electrical systems. As a result, the electrical world works primarily with models. If you can grasp the model concepts, then you can make the transition to the rest of the physical sciences and literally work any problem.

\section{RATE THE TIME}

In all the fundamental concepts, time is a denominator value. The ratio of a concept to time gives a rate. Pardon the pun, but time is a very under rated concept. Now you will always remember where time goes in the equations. rate $=1 /$ time

As would be expected from the triad principle, in the complete physical model, there are three different time based components. In some equations time is constant, so $t=1$. The second time, $t_{t}$, is associated with velocity or rate and energy. The third time, $t_{r}$, is associated with acceleration, power, and potential. The times are independent of each other in duration and direction, but they may be concurrent. 


\section{CHANGE IS NOT CONSTANT}

In the first science classes, all discussions use absolute values. In engineering, problems are presented as trends, which are differences and use calculus. In quantum physics, everything is relative. Progressively, the mathematics becomes more complex.

Actually, all the problems are the same. They are just examined from a different perspective. In absolute science, the observer is removed from the problem and is not part of the equation. As a result, numbers appear to be fixed. In differential engineering, the observer is comparing two events. As a result, one number appears to be subtracted from another. In relative physics, the observer is inside the system and he is comparing activity to his own motion. Accordingly, there is a calculation for his action and the observed action.

Time Magazine's Man of the Century, the physicist Albert Einstein, really developed the idea for relative motion [6]. This is great science for a very limited number of problems. Unfortunately, many practitioners try to shoehorn every problem into relativity. In his most famous and most understood equation, Professor Einstein succinctly attributed the mass energy conversion to an absolute value. It seems the more one understands a problem, the simpler it becomes.

Absolute relationships yield the maximum or peak value. Differential relationships yield the average performance. Relativistic relationships yield an instantaneous analysis.

In this paper, the few equations will be illustrated in an absolute format, since it is the most straightforward. However, some terms can change. Those differentiable items will be included in brackets.

For those that insist on the calculus persuasion, simply substitute a differential operator for the brackets. You will find there is no distinction in the relationships, until there is a combination of equations. Combinations will yield exponents, and the result of the differential process will yield an averaging of values. By construction, any relationship that is a differential on top, is a differential in the denominator.

\section{HOW DO YOU GET ENERGY?}

Engineering and science are often segmented into specialties or niches. However, when looking at a complete physical system, diverse analyses will arise. For example, an electrical power engineer must understand mechanical systems as well as circuits. Similarly, a controls engineer must understand chemical and thermodynamic processes as well as circuits.

So, what is the best way to blend the technologies? Energy relationships exist in all systems. Therefore, if the problem can be converted to energy $(E)$, then an equality correlation can be defined into the other sciences.

\section{TO THE POINT}

Is it possible to reduce all of the electrical and magnetic concepts into one, simple equation? What will its form be? Does it necessarily contain distance?
What concepts have been discussed so far that are related to electromagnetics? These items are charge, magnetic poles, time, and energy. In previous works, a universal equation for electromagnetic energy was proposed [2, 3]. It is restated as follows.

Electromagnetic energy is the change in the product of charge and pole strength over time.

$$
E=[p q] / t_{t}
$$

This concept is so fundamental and so inclusive that it appears intuitively obvious. However, as can be ascertained by the date of some of the early references, many years of research went into developing the concept.

This form does not contain distance. This is for point conditions that exist within any circuit. The concept of a field or spectrum is developed in a later section.

\section{MEASURE ONLY 3 THINGS}

Consider all the items that can be measured in an electrical circuit. These reduce to three practical terms voltage (v), current (i), and frequency (f). In controls, these are sometimes called the across, through, and oscillating parameters. All other concepts commonly used are actually calculated.

How do the measured values relate to the electromagnetic energy concept? They are all definitions that can be obtained from the relationship. The measured terms are rate of change or trends based on the p's and q's with time.

$$
\begin{aligned}
v & =[p] / t \\
i & =[q] / t \\
f & =1 / t
\end{aligned}
$$

In its fundamental form, frequency seems to be the rate of the unique constant, one. Actually, it is the rate of one wave, cycle, or circle.

Not only does the point form of the electromagnetic relationship contain the definition of energy, it also contains the definition of all the electric and magnetic terms that can be practically measured. That is a strong statement.

\section{CALCULATE ONLY 3 THINGS}

The measured components are unique variables, so they cannot add or subtract together. The only remaining algebraic possibilities are multiplication and division, which will create new concepts. That implies that both voltage and current would be used in the calculations.

As can be observed, voltage and current require time in the denominator. If both are used, then another unique time term ' $t_{r}$ ' is necessary, but only one time term ' $t_{t}$ ' has been included in the energy equation.

As discussed earlier, the two time terms may be coincidental, but not necessarily. Since the second time term is associated with potential, it is linked to voltage.

The product of the measured variables is power (S).

$$
S=v * i=\left[p / t_{r}\right] *\left[q / t_{t}\right]=[E] / t_{r}
$$

The ratio of the measured variables is impedance $(Z)$.

$$
Z=v / i=\left[p / t_{r}\right] /\left[q / t_{t}\right]
$$


Delay is the change in times. It is simply the difference in the time between the voltage and the current.

$$
t_{d}=t_{r}-t_{t}
$$

Depending on the specialty within the electrical community, time delay is represented in many ways. It may also be called power factor or phase shift. Phase shift is the time delay converted to angle delay between waves of the same frequency.

\section{CONSERVATION KEEPS IT IN THE FAMILY}

When there is no outside influence, a system is referred to as closed. The larger the system, the more "closed" it becomes. With this condition, the conservation of energy is defined: Energy is neither created nor destroyed, so the sum of the energy in a system is zero. [7]

Conservation is a constraint placed on problems to reduce the complexity of analysis. In actuality, there is always some outside influence, but it is often negligible.

\section{ARE THE LAWS LEGAL?}

The concepts embedded in the very fundamental electromagnetic energy equation are staggering. How many more relationships can be found? Consider just a few. Electrical energy is voltage multiplied by charge. Magnetic energy is current multiplied by magnetic pole flux.

Now the plot thickens even further. Circuit analysis is often described using two laws developed by the Prussian mathematician and physicist, Gustav Robert Kirchhoff in 1854.

Both these laws are imbedded in the very simple electromagnetic energy definition. First, apply the constraint of conservation to the relationship. This sets the sum of the energy equal to zero. Next, hold one term constant. Then, the sum of the changing term is zero.

Kirchhoff's voltage law (KVL) can be stated succinctly: When the charge is constant, the sum of the voltage around a loop is zero. [8]

$$
\begin{array}{ll}
\Sigma[p q] / t_{t}=0 \\
\Sigma v=0 & \mid q=k
\end{array}
$$

Similarly, Kirchhoff's current law $(\mathrm{KCL})$ can be stated: When the magnetic flux is constant, the sum of the current at a node is zero. [9]

$$
\begin{array}{ll}
\Sigma[p q] / t_{t}=0 \\
\Sigma I=0 & \mid p=k
\end{array}
$$

Faraday's law is also imbedded in the electromagnetic energy correlation. It states that the rate of change of the magnetic flux or poles is equal to the induced voltage. [9] Similarly, the conservation of charge states the sum of the charge is zero.[4] This is also implicit in the relationship when the constraint of conservation of energy is imposed.

The complete suite of relationships used for circuit analysis is rooted in one, very simple, elegant equation.

$$
E=[p q] / t_{t}
$$

The development and application of the concepts can most often be done with mathematics no more complex than algebra. This opens the understanding of electromagnetic science to an entire new level of application.

Another class of analysis embraces electromagnetic energy dispersed through a medium, rather than focused at a point. The investigation requires knowing the location in space. Expansion of the point form of electromagnetic energy will include distances and directions.

\section{THE FINAL FRONTIER - SPACE}

What is the most general way to describe positions and motion anywhere in the world? Is it expressed in relation to the center of the planet? That would be facetious.

Just as we live on the surface of the earth, most activity of physical systems occur on the surface of a spheroid. Hence, a ball is the best general shape to completely enclose a continuous system. A location on the surface does not have to be a uniform distance from the origin, but the shape does need to be unbroken. In essence, a route around the sphere would be a circuit.

Consider the spheroid in Figure 1. Rectangular, Cartesian, $x-y-z$ axes are the most familiar orientation for describing positions and motion. These internal references are perpendicular at the origin and are great for a square world and straight lines. They can be used as approximations for comparatively small distances on the curve. However, curvilinear motion between two points on the surface of the sphere is not well described by two ' $x-y-z$ ' positions.

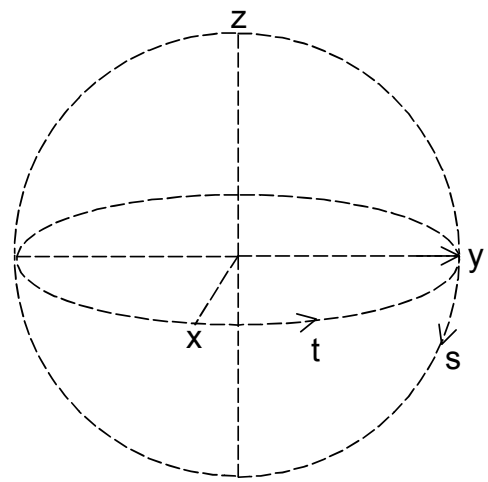

Figure 1 - Space

Electromagnetics, by nature of the physics, involves curved paths. When Captain Kirk moved the Enterprise through space, he could not describe his positions by $x-y-z$ locations. For complete circumnavigation of the globe, it is necessary to employ surface coordinates.

A global, surface system would necessarily have external axes that are perpendicular on the surface. The horizontal, or latitude, t-axis parallels the equator and portrays tangential motion. The positive direction is counterclockwise.

The vertical standing, or longitude, s-axis joins at the poles and depicts enclosing paths. Lines leave the north pole or node and enter the south pole.

The third axes is a radial along one of the internal axes, ' $x$ or $y$ '. A local origin is created on the surface where the 'st' axes intersect with the radial. 


\section{SIZE, DISTANCE, OR RAY}

When calculating volume in a space, most people revert to the absolute equations learned in early science and math classes. That is great for known shapes. However, what can be done for erratic surfaces? Can we develop an approach that is more general? It comes from vector algebra.

A vector is simply a distance that has direction. Subscripts refer to the axis that provides orientation from which the vector is measured.

As would be expected, there are three types of distances. The first is the boundary volume, space, or size represented by ' $s$ '. Tangential motion distance is represented by ' $d$ '.

The working distance to the surface from the origin is expressed with the ray ' $b y$ '. The ray is not along an axis, and the length may vary with the contour of the surface. Its length is projected on the internal, radial y-axis from which it is measured and on the arc along the standing s-axis. These vectors and axes are shown in Figure 2.

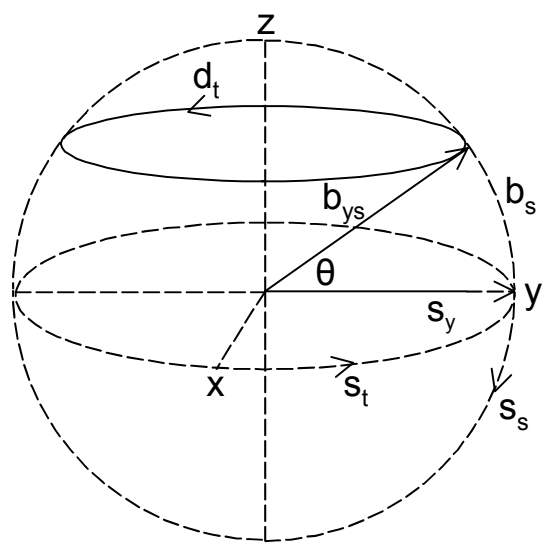

Figure 2 - Motion Space

\section{VOLUME INSIDE AND OUT}

The amount of space within the spheroid is revealed by the volume ' $V$ '. Volume is simply the product of an area ' $A$ ' and a vector distance ' $s$ ' perpendicular to its surface. Traditionally, the volume is not considered to have direction. Nevertheless, we will include direction to show the axis of the area.

Using surface vectors ' $s$ ' yields a solid area at the boundary of the spheroid. This is the total surface volume. The subscripts indicate vector rather than linear multiplication. The right hand rule and related concepts are contained in the vector orientation.

$$
V_{y}=A_{y} \bullet s_{y}=s_{s} s_{t} s_{y}
$$

Although the example refers to a specific y-axis, alternate combinations of the distances on 's-t-y' axes will yield three different areas. Nevertheless, the volume will be the same for all three.

The operational volume is calculated using the motion rather than surface vectors. Its size depends on the motion dimensions ' $b_{y s}-d_{t}-s_{y}$ ' measured from the same 's-t-y' axes as the surface volume.

$$
V_{y}=b_{y s} d_{t} s_{y}
$$

Notice that the space vector ' $s_{y}$ ' occurs in both the volumes. This will prove very significant.

\section{TIME TO SPIN}

When time is involved in the relationship, it is cyclic and causes a rotation or shift to another axis. The first time shifts to the tangential. The second time shifts to a radial $x$ or $\mathrm{y}$.

\section{FIELD OF DREAMS}

That was a relatively involved path to traverse. However, it gives the dimensional values necessary to develop a very simple electromagnetic energy field equation.

Most applications engineers will never be required to use field equations. This section is for those occasional designers that are involved with distributed systems. It will also give a basis for reading and understanding those papers that sometimes get too tedious with the Calculus.

Now what does the field relationship provide? To most engineers, the very thought of fields causes cringes and nightmares of that university class of long ago. That is because of the trauma of the mathematics associated with the dispersed electromagnetism.

We spent several paragraphs to remove the complexity of the mathematics. In determining the volume, the most difficult process used was a vector multiplication. Although it is rocket science, it is not hyperspace.

Unlike the point relationship used in circuits, fields illustrate the way electrical and magnetic properties are spread out or distributed over a volume.

Simply take the point form of the energy equation and multiply it by the motion volume in the numerator and the equivalent boundary volume in the denominator.

Phenomenally, but not very surprising, the result is the electromagnetic energy in a field. Subscripts are applied to the charge and the magnetic pole to give the axis on which they are positioned.

$$
E=\frac{\left[p_{z} \underline{q}_{y} \underline{b}_{y s} \underline{d}_{t} \underline{s}_{y}\right]}{t_{t} V_{y}}
$$

Remembering the volume definitions, it is very significant that the distance along the radial axis ' $s_{y}$ ', arises in both the numerator and denominator. This gives the direction of analysis. Obviously, the two terms can cancel to significantly reduce the complexity of many problems.

The equation shows the relative relationship of terms to each other. For analysis, orient the system so the force is along the internal ' $y$ ' axes. The ray ' $b_{y s}$ ' defines the action. If a different direction of investigation is desired for one term, simply multiply by a transformation vector.

What is the significance of this simple product of flux, charge, and distances over time? Every machines, transmission, and fields problem can be calculated from that one very simple relationship. 
You can write a very simple computer program or spread sheet to solve very complex, special problems. You will need a module to perform vector calculations and you will need to describe the shape of the surface.

\section{WHAT'S NOT THE POINT}

What are some of the concepts that are directly extracted from the distributed form of electromagnetic energy? The first obvious thing is energy, which is defined as force ' $F$ ' multiplied by the ray or lever arm distance ' $b y$ '. Therefore, force is all the terms but the ray distance. Because of the vector product associated with ' $b_{y s}$ ', torque is also derived from the force. Rotational energy is torque through an angle.

Rather than being a point used in most circuit analysis, current ' $\mathrm{I}_{t}$ ' is actually spread around the perimeter of the conductor in a phenomenon called skin effect. The perimeter is determined by the cross-sectional area ' $A_{t}$ ' as seen in Figure 3 . The current density ' $\mathrm{J}_{t}$ ' is the current over the area.

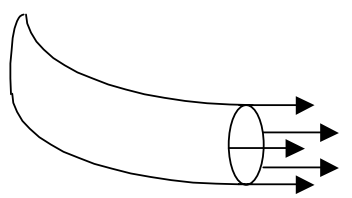

Figure 3 - Density

If current is dispersed, it is logical to expect the underlying charge ' $q y$ ' to occupy more than a point. Charge spread over the volume is called charge density ' $\rho$ '.

\section{INTENSE OR DENSE FIELD?}

We have defined the energy of a field, but how does that break down into components? Does a field relate to the voltage and current of a circuit? How are the values determined when the energy is spread out over space? Is there a difference in dynamic and static properties?

Field properties are measured relative to a dimension, either distance or area. Intensity is a measured value over a distance. Field intensity is a dynamic function since it depends on time. Density is matter over area. Energy is the product of intensity, density, and volume.

All the electrical and magnetic field equations can be extracted from the field energy relationship. Notice that the internal axes are a projection of the surface axes. For example, $s_{y}$ is a projection of $d_{t}$, and $s_{z}$ is a projection of $s_{s}$.

$$
E=\frac{\left[p_{z} \underline{q}_{y} \underline{b}_{y s} \underline{d}_{t} \underline{s}_{y}\right]}{t_{t} s_{s} s_{t} s_{y}}
$$

The electric intensity ' $E$ ' is voltage over the measurement path, ' $\mathrm{s}_{\mathrm{t}}$ '.

$$
\mathrm{E}=\left[p_{z} / t_{r} s_{t}\right]_{t}
$$

The magnetic intensity ' $\mathrm{H}$ ' is current within a closed path ' $s_{s}$ ' as seen in Figure 4.

$$
\mathrm{H}=\left[q_{y} / t_{t} s_{s}\right]_{s}
$$

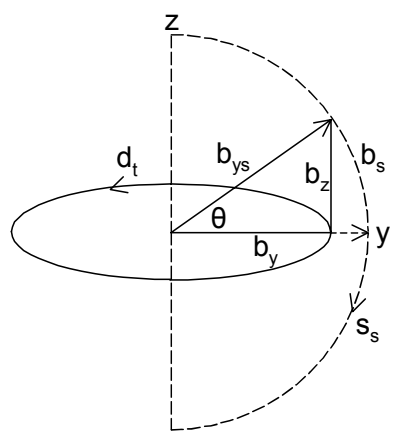

Figure 4 - Closed Path

Field density is a static function. Since it is independent of time, it must be spread over an internal area. The electric density ' $D$ ' is charge based.

$$
\mathrm{D}=\left[q_{y} / A_{y}\right]_{y}
$$

The magnetic density ' $B$ ' is magnetic strength spread over the area the lines penetrate.

$$
\mathrm{B}=\left[p_{z} / A_{z}\right]_{z}
$$

These four relationships are the foundation of all field analyses. All are contained within and extracted from the single e-m field relationship.

Energy can have either voltage or current, but not both. Calculations that have both will involve power and require the time associated with the potential voltage.

Look at what you just encountered. You have a very basic simple relationship that can do any circuit problem. You have a related equation with volume that permits the solution of all the rest of the niche problems that come up involving distributed energy. A subsequent paper addresses the impact on medicine, chemistry, biology, and other molecular problems.

\section{DON'T DO HAIRY MATH}

The next topic simply addresses some traditional math issues and the often referenced, but seldom used Maxwell equations.

Did you ever notice that you could not solve any Calculus problems directly? We are not talking about you forgot the stuff. Since you could not solve calculus, in class you set up differential equations. However, you cannot solve those either.

Why did your professors show you all that? It was a representation or model. How does anyone get an answer from a differential equation? You recognize the form and plug in values for coefficients and exponents. But that is not solving the equation in an algebraic sense.

There is another thing to keep in mind about calculus and differential equations and all that kind of math. The most complex problem in physical science reduces to a second order differential equation. In other words, one component does not have time, one component is rate, and one component is acceleration. Since we know that all physical problems fit that structure, we now know the form for all answers to a differential equation for physical systems. 
If all the differential discussion was not overwhelming enough, someone came along with the idea of partial differential equations. Again, in physical realizations, that reduces to a three directional structure. Partials are actually the component of an equation broken down into the $x-y-z$ component directions.

\section{THIS WILL CURL YOUR HAIR}

When the Scottish mathematician, John Clerk Maxwell, set up his four equations in 1873, he was attempting to make everything in a simple structure. Although his basic premise was an excellent idea, his approach let the math overload the comprehension of the concepts.

The Professor used a rather arcane approach to his ideas. He employed the concept of a divergence and a curl. What is that?

Well he defined a three-dimensional partial derivative as a del. He appropriately used the obscure Egyptian nabla symbol ' $\nabla$ ', to represent the complex math operator. Then, curl is the cross product of the del with field intensity. Divergence is the dot product of the same 3-D directions with density. $[10,11]$

Since we will not use the equations, we will not clutter up your reading with the intricacies and the space of the calculations.

For that matter, do you even remember Maxwell's equations? The equations are seldom used but the name is invoked to introduce a concept. Nevertheless, the acknowledgement of the suite of equations is accepted in passing by every electrical engineer and physicist. However, like other applications engineers, we will skip over the equations. Nevertheless, for your convenience of reference, they will be listed in a cleaned up form in the appendix.

Even with all the convoluted operations, the results of these equations do not inherently show the directions of the fields. So those are some of the reasons Maxwell and the del are seldom used. The math just becomes too complex and the meaning is obscured from normal applications.

\section{MAXED OUT}

It is necessary to refer to the four equations, so that we can illustrate a simpler solution based on the field form of energy. This one equation contains all the info of Maxwell, without the math.

By the structure of the vectors, it is unnecessary to apply the partial derivative operator. Maxwell's del can be replaced by Durham's ' $1 / \mathrm{s}_{\mathrm{y}}$ '. The result is an algebraic operation, rather than partial derivatives.

To illustrate that, take each of the field properties described earlier and multiply it by ' $1 / \mathrm{s}_{\mathrm{y}}$ '. The result is an algebraic equivalence to the calculus of the del. Some engineers want to work an example to help in their understanding. Several calculus and partial derivative relationships are included in the appendix for those that want to do the mathematical exercise.

Maxwell was describing all electromagnetic activity compared to the origin of a Cartesian system ' $s_{x}, s_{y}, s_{z}$ '. By changing perspective to a surface analysis ' $s_{t}, s_{s}, s_{y}$ ', the complex partial derivative study is unnecessary, since all the associations are in a simple product.

\section{CONCLUSIONS}

So what is the deal? Electro-magnetics is made up of electrical charges and magnetic poles moving in some time frame.

$$
E=[p q] / t_{t}
$$

The circuit or rotational motion makes a sphere. Simply by maintaining the directional orientation, it is possible to identify all fields problems from one very simple algebraic relationship.

$$
E=\frac{\left[p_{z} \underline{q}_{y} \underline{b}_{y s} \underline{d}_{t} \underline{s}_{y}\right]}{t_{t} V_{y}}
$$

The challenge then becomes determining which terms you want to use. One equation can describe all the electromagnetic analysis. The complete model includes fields and dispersion in space. When the distances are resolved, the relationship simplifies to a circuit problem.

\section{ACKNOWLEDGEMENTS}

A large part of the success of any author's effort is the extensive peer review process that the PCIC encourages. Numerous people made very valuable comments for explaining this simple concept.

\section{REFERENCES}

[1] Fazarine, Zvonko, "A Viewpoint on Calculus," HewlettPackard Journal, Palo Alto, CA, June, 1987. Previously presented to Mathematics Panel of American Association for the Advancement of Science.

[2] Durham, Marcus O., "A Composite Approach to Electrical Engineering", Institute of Electrical and Electronic Engineers Region V, 88CH25617-6/000143, Colorado Springs, CO, March 1988, pp 143-147.

[3] Durham, Marcus O., "A Universal Systems Model Incorporating Electrical, Magnetic, and Biological Relationships," IEEE Transactions on Industry Applications, Vol. 29, No. 2, March/April 1993, pp 436446.

[4] Semat, Henry, and Robert Katz, Physics, Rinehart \& Co., New York, 1958.

[5] Wher, M. Russell, James A. Richards, and Thomas W. Adair III, Physics of the Atom, Addison-Wesley, Ready, MS, 1978.

[6] Einstein, Albert, Relativity - the Special and the General Theory, authorized translation by Robert W. Lawson, Crown Publishers, New York, 1961.

[7] Van Wylen, Gordon J., Thermodynamics, John Wiley, New York, 1964. 
[8] Chirlian, Paul M., Basic Network Theory, McGraw-Hill, New York, 1969.

[9] Chapman, Stephen J., Electric Machinery Fundamentals, McGraw-Hill, New York 1985.

[10] Hoyt, William H., Engineering Electromagnetics, McGraw-Hill, New York, 1958.

[11] Johnk, Carl T. A., Engineering Electromagnetic Fields and Waves, John Wiley, 1988.

[12] Taylor, Angus E., Calculus with Analytic Geometry, Prentice Hall, Englewod Cliffs, New Jersey, 1963.

\section{APPENDIX A STAR OF THE SHOW}

Now that a technique has been illustrated for defining space, can all this be placed into one mathematical structure? One effort is often used. The Swiss mathematician Leonhard Euler explained the calculus in terms of functions in 1748. One of his many definitions explains that the natural base 'e' raised to the power of an angle is the vector sum of the sine and the cosine of the angle. [12]

Euler's rule is great for rectangular motion, but like the internal axes on which it is based, it is limited in ability to portray the track of curve motion.

A star product $\left({ }^{*}\right)$ is proposed to describe the global, surface motion. This includes a combination of the cross and dot product into one term with a ray vector representing curve motion.

$$
\begin{aligned}
1_{r} * b_{r s} & =\left(1_{r} \bullet b_{r}\right)_{r}+\left(1_{r} \times b_{s}\right)_{t} \\
& =\left(1_{r} b_{r}\right)_{r}+\left(1_{r} b_{z} \theta_{r s}\right)_{t}
\end{aligned}
$$

The radial $r$-axis can be either the $x$ or $y$-alignment. The star product permits one expression to represent both linear energy and rotational energy including torque. Anytime the ray vector 'b' arises, it implies a star product, since it has a double subscript. The separation of the distances are shown in Figure 5.

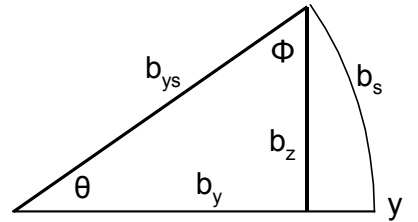

Figure 5 - Vectors

\section{APPENDIX B MAXWELL CORRELATION}

The suite of equations developed by Maxwell contains four relationships.

$$
\begin{array}{ll}
\nabla \times E=-d B / d t & \text { Volt } / m^{2} \\
\nabla \times H=J+d D / d t & \text { Amp } / m^{2}
\end{array}
$$

$$
\begin{array}{ll}
\nabla \cdot \mathrm{D}=\rho & \mathrm{Cb} / \mathrm{m}^{3} \\
\nabla \bullet \mathrm{B}=0 &
\end{array}
$$

Using the common internal, radial vector ' $1 / \mathrm{s}_{\mathrm{y}}$ ', rather than the del, the suite of four equations can be calculated from the single unified electric-magnetic energy field relationship.

$$
E=\frac{\left[p_{z} \underline{q}_{y} \underline{b}_{y s} \underline{d}_{t} \underline{s}_{y}\right]}{t_{t} V_{y}}
$$

First the intensity or density relationship will be shown as previously defined. Next, to obtain volumetric terms, both sides of the equation will be multiplied by the inverse of the vector along the $y$-axis, ' $1 / s_{y}$ '. The subsequent equations manipulate the vector algebra. The result is a relationship that is equivalent to one of the del equations.

This simple process uses a unified electromagnetic equation with a vector along an axis. This eliminates the complex calculus of Maxwell in exchange for a simple algebra operation.

Intensity: The distances we have used in the dynamic or intensity relationships are relative to the external reference axes ' $s_{t}, s_{s}, s_{y}$ '. These inherently contain the cross product of the del ' $\nabla$ '. The vector in the radial direction ' $s_{y}$ ' multiplied by a vector on the surface yields an area in the other surface direction.

Equation of electric intensity

$$
\begin{aligned}
& \mathrm{E}_{\mathrm{t}}=\left[p_{z} / t_{t} \mathrm{~s}_{t}\right]_{t} \text { Volt } / \mathrm{m} \\
& \begin{array}{rlr}
\left(1 / \mathrm{s}_{y}\right) \mathrm{E}_{\mathrm{t}} & =\left[p_{z} / t_{t} s_{y} s_{t}\right] \quad \text { Volt } / \mathrm{m}^{2} \\
& =\left[p_{z}\right] / t_{t} A_{-s} \\
& =\left[\mathrm{B} / t_{t}\right]_{-s}=-\left[\mathrm{B} / t_{t}\right]_{s} \\
& =\nabla \times \mathrm{xE}
\end{array}
\end{aligned}
$$

Equation of magnetic intensity

$$
\begin{aligned}
& \begin{array}{ll}
\mathrm{H}_{\mathrm{s}}=\left[q_{y} / t_{t} \mathrm{~s}_{s}\right]_{s} & \mathrm{Amp} / \mathrm{m}
\end{array} \\
& \left(1 / s_{y}\right) H_{s}=\left[q_{y} / t_{t} s_{y} s_{s}\right] \quad \mathrm{Amp} / \mathrm{m}^{2} \\
& =i / A_{t} \\
& =J_{\mathrm{t}} \\
& =\nabla \times H
\end{aligned}
$$

$$
\begin{aligned}
& \text { Equation of charge density } \\
& \qquad \begin{array}{rlr}
\mathrm{D}_{\mathrm{y}}=\left[q_{y} / A_{y}\right] & \mathrm{Cb} / \mathrm{m}^{2}
\end{array} \\
& \qquad \begin{array}{rlr}
{\left[\mathrm{D}_{\mathrm{y}} / t_{t}\right]} & =\left[q_{y} / t_{t} A_{y}\right] & \mathrm{Amp} / \mathrm{m}^{2} \\
& =i / A_{y} & \\
& =\mathrm{J} & \\
& =\nabla \times \mathrm{H}
\end{array}
\end{aligned}
$$

Density: the distances in the static or density relationships are relative to the internal, reference axes ' $s_{x}$, $s_{y}, s_{z}$ '. These inherently contain the dot product of the del ' $\nabla$ '.

The vector in the radial direction ' $s_{y}$ ' multiplied by the plane area in the direction of the displacement yields a volume. In the magnetic equation, the radial and the plane area are in different directions. Hence, the result of a dot product in two different directions does not exist. 


$$
\begin{aligned}
& \text { Equation of electric density } \\
& \mathrm{D}_{\mathrm{y}}=\left[q_{y} / A_{y}\right] \\
& \mathrm{Cb} / \mathrm{m}^{2} \\
& \left(1 / s_{y}\right) D_{y}=q_{y} / A_{y} s_{y} \\
& =q_{y} / V_{y} \\
& =\rho_{\mathrm{y}} \\
& =\nabla \bullet \mathrm{D}
\end{aligned}
$$

Equation of magnetic density

$$
\begin{array}{rlr}
\mathrm{B}_{\mathrm{z}}=\left[p_{z} / A_{\mathrm{z}}\right] & \mathrm{Wb} / \mathrm{m}^{2} \\
\mathrm{~B}_{\mathrm{z}} / \mathrm{s}_{y} & =p_{z} / A_{z} \mathrm{~s}_{y} & \mathrm{~Wb} / \mathrm{m}^{3} \\
& =0 & \\
& =\nabla \bullet \mathrm{B} &
\end{array}
$$

It is fascinating that all the action is on the radius axis ' $s y$ '. However, it is the understanding of physical relationships that make the unified electric-magnetic equations possible.

\section{VITAE}

Marcus O. Durham is the Principal Engineer of THEWAY Corp, Tulsa, OK. He is also a Professor at the University of Tulsa and he is President of Advanced Business Technology, an entrepreneurial firm in the emerging ecommerce arena.

$\mathrm{He}$ is a registered Professional Engineer, a state licensed electrical contractor, a FCC licensed radiotelephone engineer, and a commercial pilot. Professional recognition includes Fellow of the Institute of Electrical and Electronic Engineers, Diplomate of American College of Forensic Examiners, member of the Society of Petroleum Engineers, and task group member of American Petroleum Institute. He has been awarded the IEEE Richard Harold Kaufmann Medal "for development of theory and practice in the application of power systems in hostile environments." He was recognized with 3 IEEE Awards for his Standards development work. He has been awarded numerous times for the over 100 technical papers he has authored. He is acclaimed in Who's Who of American Teachers, National Registry of Who's Who, Who's Who of the Petroleum and Chemical Industry of the IEEE, Who's Who in Executives and Professionals, Who's Who Registry of Business Leaders, Congressional Businessman of the Year, and Presidential Committee Medal of Honor. Honorary recognition includes Phi Kappa Phi, Tau Beta Pi, and Eta Kappa Nu.

Dr. Durham received the B.S. in electrical engineering from Louisiana Tech University, Ruston; the M.E. in engineering systems from The University of Tulsa, Tulsa, OK; and the Ph.D. in electrical engineering from Oklahoma State University, Stillwater.

Robert A. Durham received a Bachelors of Science degree in Electrical Engineering from the University of Tulsa in 1992. He earned a Masters degree in Engineering Management from the University of Tulsa in 1997. He is a registered Professional Engineer in Oklahoma and Texas.

His technical emphasis has been on all aspects of power systems from electric generating stations, to EHV transmission systems, to large-scale distribution systems and power applications for industrial locations. He is a nationally recognized author; having received several awards from technical and professional organizations such as the IEEE, and has published magazine articles on multiple occasions.

Currently, Robert is the principal of D2 Technologies. Previously he was Manager of Electric Infrastructure for an oil and gas exploration and development company, and was in Planning and Project Management for a major Utility. $\mathrm{He}$ is also President of the Organization for Entrepreneurial Management, an internet-based firm specializing in traffic development for consumer sites.

Karen D. Durham received a Bachelors of Science degree in Chemical Engineering from the University of Tulsa in 1997. Her technical emphasis has been on design, research, and system modeling. She has researched environmental remediation and reclamation of oil-impacted soils. She has worked in the design of flares, incinerators, and burners for disposal of waste products.

She has designed and overseen the construction of research equipment, and she has led investigations for developing and modifying oil and gas separation equipment. Also, she has written Visual Basic computer models of separation and heat transfer equipment. She has published papers on bioremediation, phytoremediation, voltage suppression, and system modeling.

Karen is currently a Development Engineer for NATCO, an oil and gas processing equipment company based in Houston, Texas and is a Director with Advanced Business Systems, specializing in entrepreneurial Internet commerce. 